\title{
Emission properties and temporal coherence of the dark exciton confined in a GaAs/ $\mathrm{Al}_{x} \mathrm{Ga}_{1-x} \mathrm{As}$ quantum dot
}

\author{
S. Germanis $\odot,{ }^{*}$ P. Atkinson, R. Hostein, S. Majrab, F. Margaillan $\odot$, M. Bernard, V. Voliotis $\odot$, and B. Eble $\odot^{\dagger}$ \\ Sorbonne Université, CNRS, Institut des Nanosciences de Paris, 75005 Paris, France
}

(Received 2 April 2021; revised 5 September 2021; accepted 8 September 2021; published 16 September 2021)

\begin{abstract}
We report measurements of the radiative lifetimes and coherence times of the dark and bright excitons in an asymmetric GaAs/AlGaAs quantum dot. The dots, fabricated by partial infilling of asymmetric in situ etched nanoholes, have low symmetry, which leads to significant dark-bright mixing as demonstrated by dark-bright anticrossing in magnetophotoluminescence spectra. Using an orthogonal excitation-detection waveguiding geometry and quasiresonant excitation, we compare the coherence properties, measured by Michelson interferometry, of the dark and bright exciton from the same dot in the absence of an external magnetic field.
\end{abstract}

DOI: 10.1103/PhysRevB.104.115306

\section{INTRODUCTION}

The low emission of dark excitons (DEs) in confined semiconductor quantum dots (QDs) can be easily detected nowadays thanks to high spatial resolution spectroscopy, resonant fluorescence experiments, and coupling to photonic cavities [1]. Due to spin conservation rules, a DE that is formed by one electron and one hole with parallel spins cannot absorb or emit a photon. However, its weak coupling to light as reported in strained InAs QDs has enabled the demonstration of a very long spin coherence time of $\sim 100 \mathrm{~ns}$ [2] compared to $\sim 300 \mathrm{ps}$ for the bright exciton (BE) [3]. Moreover, similar to a hole spin [4,5], a DE is rather insensitive to nuclear spin noise [6]. Recently, a DE was proposed as an intermediate state in the generation of photon cluster states [7] showing that the DE can be considered as a coherent electronic excitation and a promising solid-state qubit. Therefore, several new experimental strategies have been proposed to selectively increase the generation rate of the DE in QDs [8-11].

The valence-band mixing leading to the brightening of the DE in InAs/GaAs and CdTe/ZnTe QDs in the absence of an externally applied magnetic field is known to be caused by a reduction in the dot symmetry due to the anisotropic strain field that is natively present in such dots [12]. Here, we focus instead on the mechanisms leading to the DE emission from a virtually strain-free $\mathrm{GaAs} / \mathrm{Al}_{x} \mathrm{Ga}_{1-x} \mathrm{As} \mathrm{QD}$ grown by nanohole infilling. The emission of the $\mathrm{DE}$ was previously reported using highly symmetric GaAs/AlGaAs QDs located close to the cleaved edges of the sample, where the residual strain created by relaxation at the cleaved edge was estimated at $\epsilon \sim 0.1 \%$, and it played a major role [13]. In contrast, here we detect emission of the DE from asymmetric $\mathrm{GaAs} / \mathrm{Al}_{x} \mathrm{Ga}_{1-x}$ As QDs deeply embedded in a homogeneous

\footnotetext{
*germanis@insp.jussieu.fr

†benoit.eble@insp.jussieu.fr
}

GaAs matrix. The magnitude of the residual strain is expected to be at least one order of magnitude smaller, i.e., $\epsilon \sim 0.01 \%$, based on previously reported optically detected magnetic resonance-like measurements on similar dots [14]. This indicates that shape anisotropy is sufficient to drive brightening of the DE in intrinsically low-strain dots.

In the following, we will examine the two distinct processes leading to the DE emission perpendicular and parallel to the growth axis describing the link between the morphological properties of the QDs and the DE luminescence. To characterize the mechanism leading to $\mathrm{DE}$ emission parallel to the growth axis, we use magneto-PL spectroscopy in the Faraday configuration.

Finally, the brightening of the DE, even weak, allows us to directly measure its emission dynamics. In particular, we present a measurement of the coherence loss of the DE state, and we compare it to that of the BE of the same QD.

\section{BRIGHTENING OF THE DARK EXCITON IN A STRAIN-FREE QD GROWN ALONG THE [001] DIRECTION}

\section{A. General considerations}

Strain anisotropy, shape anisotropy, and atomic disorder at a heterointerface all lead to symmetry breaking of the confined eigenstates of a single QD. The effect of this symmetry breaking primarily affects the valence states, and it can be described by the valence-band-mixing (VBM) parameters $\beta$ and $\gamma$. Taking into account the admixture with the light-hole (LH) bands, the expression of the lowest-energy heavy-hole $(\mathrm{HH})$-like states with which the electron-hole $(e-h)$ pair is made can be written as

$$
\begin{aligned}
\langle\mathbf{r} \mid 3 \widetilde{2,+3} / 2\rangle= & \chi_{\mathrm{hh}}(\mathbf{r}) u_{+3 / 2}(\mathbf{r}) \\
& +\chi_{\mathrm{lh}}(\mathbf{r})\left[\beta u_{-1 / 2}(\mathbf{r})+\gamma u_{+1 / 2}(\mathbf{r})\right]
\end{aligned}
$$


and

$$
\begin{aligned}
\langle\mathbf{r} \mid 3 / \widetilde{2,-3} / 2\rangle= & \chi_{\mathrm{hh}}(\mathbf{r}) u_{-3 / 2}(\mathbf{r}) \\
& +\chi_{\mathrm{lh}}(\mathbf{r})\left[\beta^{\star} u_{1 / 2}(\mathbf{r})-\gamma^{\star} u_{-1 / 2}(\mathbf{r})\right],
\end{aligned}
$$

where $\chi_{\mathrm{hh} / \mathrm{h}}$ are the envelope wave functions of the $\mathrm{HH}$ and $\mathrm{LH}$, and $u_{j}$ are the Bloch functions at the $\Gamma$ point associated with the band $|3 / 2, j\rangle$. The optical matrix elements relative to the DE take the form $\left\langle 0\left|A_{i} \hat{p}_{i}\right| \mathrm{DE}\right\rangle$, where $|0\rangle$ is the vacuum state, $\vec{A}$ is the potential vector, and $\hat{p}$ is the momentum operator. As pointed out in Ref. [15], this quantity is null for $i=x, y$ and proportional to $\sqrt{\frac{2}{3}} \beta\left\langle\chi_{e} \mid \chi_{\mathrm{lh}}\right\rangle$ for $i=z$, where $\chi_{e}$ is the electron envelope wave function. This shows that the VBM parameter $\beta$ opens a dipole allowed transition for the $\mathrm{DE}$, only perpendicular to the growth direction. We note that $\gamma$ plays no role in this DE edge-emission brightening.

However, this mechanism cannot explain the luminescence of the dark state along the growth axis, as already reported in Ref. [2]. The brightening of the DE along the growth direction is based on a dark-bright exciton coupling via $e-h$ exchange interaction. This requires a strong reduction of the symmetry to $C_{s}$ symmetry, for which the twofold rotational invariance is lost, while only a single mirror symmetry containing the growth axis remains [16]. The dark-bright mixing Hamiltonian can be rewritten as $[17,18]$

$$
\begin{aligned}
\delta \hat{H}_{C_{s}}= & \frac{\Delta_{11}}{2}(|+1\rangle\langle+2|-|-1\rangle\langle+2|) \\
& +\frac{\Delta_{12}}{2}(|+1\rangle\langle-2|-|-1\rangle\langle-2|)+\text { H.c. }
\end{aligned}
$$

where $| \pm 1\rangle=\left|s_{z}^{e}=\mp 1 / 2 ; 3 / \widehat{2, \pm 3} / 2\right\rangle$ and $| \pm 2\rangle=\mid s_{z}^{e}=$ $\pm 1 / 2 ; 3 \longdiv { 2 , \pm 3 } / 2\rangle$. In the framework of the effective-mass approximation and only taking into account Coulomb correlations for the lower-energy single-particle states, we have previously shown that the amplitudes of the dark-bright exciton mixing terms $\Delta_{11}$ and $\Delta_{12}$ are proportional to the magnitude of the VBM parameter $\gamma$ [17]. We have also studied the effect of the dark-bright exciton coupling on excited multicharged excitonic complexes whose dark spin configurations have shown a detectable level of absorption [19]. On this occasion, we were able to give an estimation of the amplitude of the dark-bright exciton mixing terms - a few tens of $\mu \mathrm{eV}$-based on the difference in absorption between the dark and bright spin configurations. We show here a direct measurement of these quantities.

In InAs/GaAs QDs and CdTe/ZnTe QDs, biaxial strain and shear strain dominate significantly to the magnitude of the VBM parameters $\beta$ and $\gamma$, respectively $[12,17]$. In the case of strain-free QDs, several mechanisms have been proposed to explain the VBM observed with no single mechanism dominating the magnitude of the VBM. For example, for GaAs/AlGaAs QDs it was shown that atomistic-level asymmetries lead to the VBM, such as the local symmetry of the interfaces between the dot and barrier materials as well as the intrinsic $C_{2 v}$ crystal-field effect. These atomistic considerations lower the symmetry of the heterostructure down to the $C_{2 v}$ symmetry, even if the geometry of the potential holds a high $D_{2 d}$ symmetry, giving rise to significant LH admixture values [20]. If in addition these GaAs/AlGaAs QDs are flat, (a) (b)

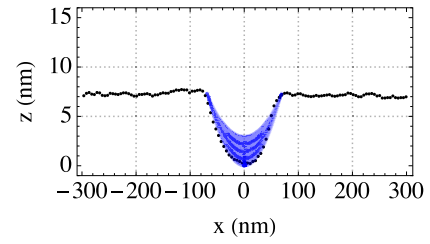

(c)

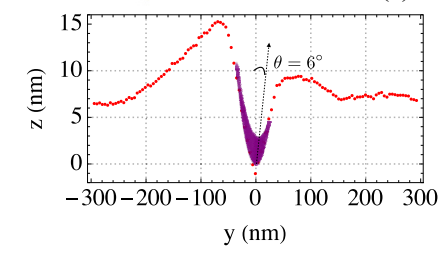

FIG. 1. (a) AFM image of a typical nanohole before infilling. (b),(c) Profiles of the nanohole along the two perpendicular crystallographic directions, [110] and [110], respectively. The infilling of the nanohole leads to lowering the QD potential down to the $C_{s}$ symmetry. Also shown are estimated QD shapes following the procedure described in Fig. 3, with a QD height of $3 \mathrm{~nm}$, an average radius $R_{\mathrm{QD}}=45 \mathrm{~nm}$, and an ellipticity $\epsilon=0.52$.

i.e., when the typical lateral size is greater than the height, in-plane distortion of the geometrical potential such as an elongation along a crystallographic direction also yields a minor contribution to the VBM [21]. However, we will show by simple considerations that an out-of-plane distortion reduces the confinement potential symmetry to $C_{s}$ and becomes a dominant factor determining the magnitude of the valence-band mixing, allowing the brightening of the DE to be linked to the geometry of the QD.

Based on AFM microscopy, we will show that GaAs/AlGaAs QDs fabricated by infilling of asymmetric etched nanoholes can have the morphological properties suitable for the DE brightening.

\section{B. Sample description}

The GaAs/AlGaAs QDs measured here were fabricated by molecular beam epitaxy on GaAs (001) substrates. Lowdensity nanoholes were etched in situ by depositing excess group III metal, which forms droplets on the surface, followed by annealing under an arsenic flux [22]. Competition between dissolution of the substrate under the gallium or aluminum droplet, out-diffusion from the droplet to the surrounding area, and recrystallization of the liquid droplet result in a nanohole surrounded by mounds formed from recrystallized residues of the original droplet $[19,22-24]$ as shown by the AFM images in Fig. 1(a).

Two different structures have been studied. Sample A used nanoholes etched by Ga droplets deposited on a GaAs buffer layer at a substrate temperature of $520^{\circ} \mathrm{C}$ [23]. The droplet deposition amount was $3 \mathrm{ML} \mathrm{Ga}$ at a flux equivalent that was used for $0.25 \mathrm{ML} / \mathrm{s}$ GaAs. A 5 min growth interruption under arsenic after droplet deposition results in nanoholes 8-10 nm deep, with an asymmetric profile [see Fig. 1(a)] and complete recrystallization of the droplet material into GaAs. These nanoholes were infilled by a $7 \mathrm{~nm} \mathrm{Al}_{0.44} \mathrm{Ga}_{0.66} \mathrm{As}$ 
lower barrier, a $0.75 \mathrm{~nm} \mathrm{GaAs}$ followed by a 3 min growth interruption under arsenic flux, a $70 \mathrm{~nm} \mathrm{Al}_{0.375} \mathrm{Ga}_{0.625}$ As upper barrier, a $20 \mathrm{~nm} \mathrm{Al}_{0.44} \mathrm{Ga}_{0.66}$ As cladding layer, and a $10 \mathrm{~nm} \mathrm{GaAs}$ cap. The 3 min growth interruption ensured net migration of GaAs towards the bottom of the nanohole after the growth of the $0.75 \mathrm{~nm} \mathrm{GaAs}$ layer, resulting in inverted dots with height $\sim 5 \mathrm{~nm}$ (see Fig. 1).

Sample B consisted of dots embedded in the middle of a waveguide structure for use in resonant excitation experiments where an orthogonal excitation-detection measurement scheme was used. In this case, the dots were located in the middle of a 400-nm-thick $\mathrm{Al}_{0.33} \mathrm{Ga}_{0.67}$ As layer, with 1 micron $\mathrm{Al}_{0.70} \mathrm{Ga}_{0.30} \mathrm{As}$ top and bottom cladding layers. To define the dots, nanoholes were etched by $\mathrm{Al}$ droplet deposition on the $\mathrm{Al}_{0.33} \mathrm{Ga}_{0.67} \mathrm{As}$ surface at a substrate temperature of $560^{\circ} \mathrm{C}$ [23]. The droplet deposition amount was 1.5 ML AL at a flux equivalent to that used for $0.3 \mathrm{~mL} / \mathrm{S}$ AlAs. A 3 min growth interruption under arsenic results in nanoholes approximately 12-14 nm deep, with an asymmetric profile similar to sample A. The nanoholes were then infilled by a thin, $2 \mathrm{~nm} \mathrm{AlAs}$ barrier, $1 \mathrm{~nm} \mathrm{GaAs}$ followed by a $15 \mathrm{~s}$ growth interruption resulting in inverted dots with a height $\sim 4-6 \mathrm{~nm}$. The dots were capped by a $2 \mathrm{~nm}$ AlAs top barrier followed by the growth of the upper half of the $\mathrm{Al}_{0.33} \mathrm{Ga}_{0.67} \mathrm{As}$ waveguide core and the upper cladding layer.

In both cases, the nanohole density was $0.4 \mathrm{dots} / \mu \mathrm{m}^{2}$, and the nanoholes had an asymmetric shape that persisted after overgrowth by a thin barrier layer, as can be seen by the line profiles along the [110] and [1 $\overline{1} 0]$ directions shown in Figs. 1(b) and 1(c). For both samples A and B, the resultant dot heights measured by AFM of uncapped infilled nanoholes was around 4-6 nm, in good agreement with the dot emission energy (see Fig. 1). However, the ensemble emission linewidth was narrow, $\sim 10 \mathrm{meV}$ for both samples, corresponding to a spread in dot height of only a few GaAs monolayers, i.e., much smaller than $+/-1 \mathrm{~nm}$ variation in nanohole depth measured by AFM. This is due to the fact that the nanoholes are only partially infilled, which leads to a spread in dot height similar to the spread in width of a GaAs quantum well [23]. The waveguide sample B showed a greater incidence of charged excitons due to the high aluminum content in the sample and impurities in the aluminum source at the time of growth. Apart from this, no significant difference in the PL characteristics-emission energy, fine-structure splitting of the neutral exciton, range of $\beta$ values, or incidence of optically active DE-was observed between the two samples.

The AFM profiles shown in Figs. 1(b) and 1(c) clearly establish the loss of the twofold rotation symmetry: the profile taken along the [110] direction is symmetric, whereas for the profile along [1히, which has the smallest lateral size, the slopes of both sides of the nanohole are asymmetric and differ from the [110] direction. These observations demonstrate a geometrical confinement potential $V_{\mathrm{QD}}(\mathbf{r})$ having a single mirror symmetry with a $C_{S}$ symmetry.

This nanohole asymmetry is due to two factors. First, a difference in As bonding configuration along the [110] and [110] directions [25] leads to an increased etching rate and hence a hole width along the [110] direction approximately two times larger than the width along the [110] direction. Secondly, droplet motion occurring along the [110] direction
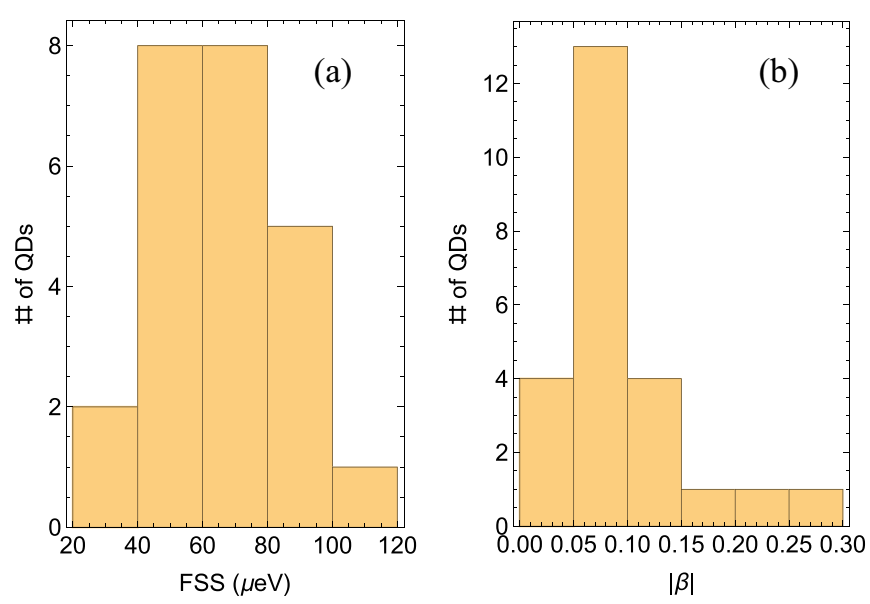

FIG. 2. Occurrence histogram of (a) the fine-structure splitting (FSS) of the neutral bright exciton, and (b) the VBM parameter, $|\beta|$, obtained by measuring the linear polarization diagram, for $25 \mathrm{QDs}$ in sample A.

during the droplet etching and recrystallization process [26] leads to an additional asymmetry in the hole profile along the [11̄0] direction also.

Figure 2 shows histograms of the fine-structure splitting (FSS) of the neutral bright exciton and the VBM parameter, $\beta$, demonstrating the lack of dot symmetry. The distribution of the neutral (bright) exciton FSS presented in Fig. 2(a) shows that a significant number of QDs have a FSS $\geqslant 80 \mu \mathrm{eV}$. This suggests a strong shape anisotropy, as indicated by the AFM profiles in Fig. 1. This is confirmed by the $\beta$ distribution in Fig. 2(b) obtained from measurements of the polarization diagrams of the BEs under off-resonant laser excitation. It gives a mean value of $\beta \sim 0.09$, similar to the distribution observed for strained InAs/GaAs QDs [27].

Based on these observations, we present next a model describing the evolution of the VBM magnitude as a function of the reduced QD symmetry.

\section{Calculation of the VBM parameters in a $C_{s}$ symmetry potential: A toy model}

\section{Modeling of the geometrical potential $\hat{V}_{Q D}(\mathbf{r})$}

The asymmetric QD shape is modeled by applying simple geometrical transformations to a $D_{2 d}$ potential, which is defined as the intersection of two spheres as shown in Fig. 3(a). The volume of intersection determines the central height of the $\mathrm{QD} h_{\mathrm{QD}}$, the radius $R_{\mathrm{QD}}$, as well as the height at the hole edge, $h_{2}$ [see Fig. 3(a)].

(i) The lateral shape of the QD is modified by applying a scaling transformation $x^{\prime}=x(1+\epsilon)$ and $y^{\prime}=y /(1+\epsilon)$, with $\epsilon$ being the in-plane anisotropy parameter, and $x(y)$ is the major (minor) axis of the QD parallel to the [110] ([110]) direction [28] [Fig. 3(b)].

(ii) Finally, the out-of-plane anisotropy is taken into account by rotating by an angle $\theta$ the QD along the axis parallel to [110] passing through the bottom of the nanohole [Fig. 3(c)]. This operation does not alter the profile along the [110] direction, and it is able to reproduce the profile along 


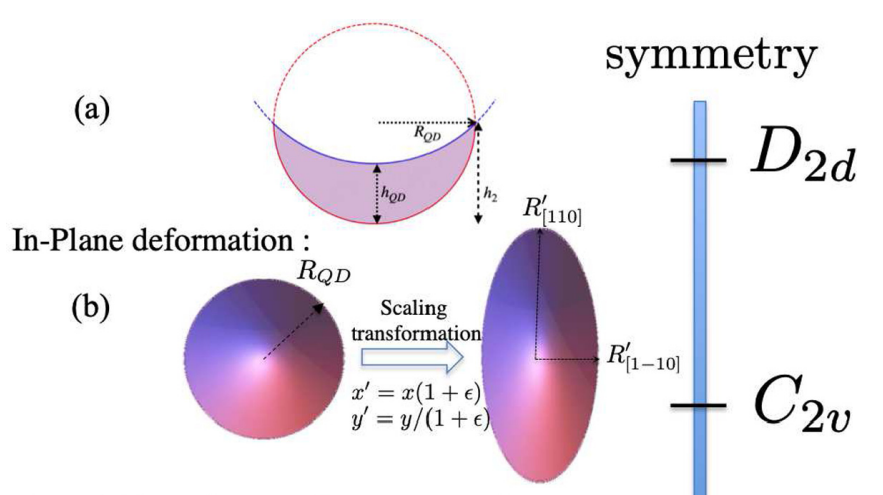

Out-of-Plane deformation :

(c)
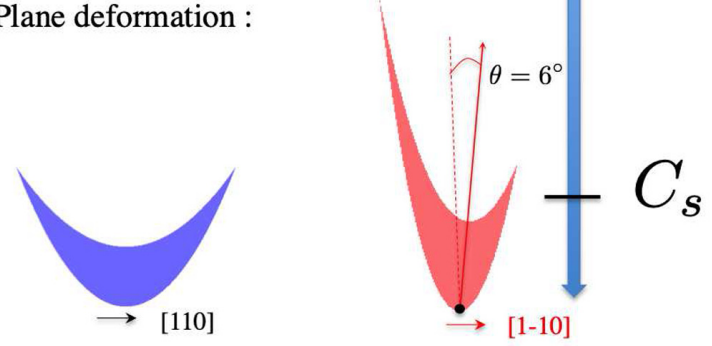

FIG. 3. Description of the different steps of designing a $C_{s} \mathrm{QD}$ from a $D_{2 d}$ shape, compatible with the anisotropic profile of the nanohole. (a) Quantum dot with $D_{2 d}$ symmetry created by the intersection of two spheres. The height and radius of the QD are defined as $h_{\mathrm{QD}}$ and $R_{\mathrm{QD}}$; the initial hole depth is $h_{2}$. (b) In-plane deformation to model the anisotropic shape of the nanohole along [110] and [110], where $\epsilon$ is the in-plane anisotropy parameter. (c) Out-of-plane deformation to model the anisotropic profile of the nanohole along the $[1 \overline{1} 0]$ direction due to droplet motion during etching.

the [110], as shown in Fig. 1(c). The angle $\theta$ provides thus the out-of-plane anisotropy.

The VBM parameters are deduced from the diagonalization of the $4 \times 4$ Luttinger Hamiltonian expressed in the basis $\left\{\left|u_{3 / 2}\right\rangle,\left|u_{1 / 2}\right\rangle,\left|u_{-1 / 2}\right\rangle,\left|u_{-3 / 2}\right\rangle\right\}$ :

$$
\left(\begin{array}{cccc}
E_{\mathrm{hh}} & -\left\langle S_{k}\right\rangle & \left\langle R_{k}\right\rangle & 0 \\
-\left\langle S_{k}\right\rangle^{\star} & E_{\mathrm{lh}} & 0 & \left\langle R_{k}\right\rangle \\
\left\langle R_{k}\right\rangle^{\star} & 0 & E_{\mathrm{lh}} & \left\langle S_{k}\right\rangle \\
0 & \left\langle R_{k}\right\rangle^{\star} & \left\langle S_{k}\right\rangle^{\star} & E_{\mathrm{hh}}
\end{array}\right),
$$

where $\quad E_{\mathrm{hh}}=\left\langle 3 / 2,3 / 2\left|\hat{P}_{k}+\hat{Q}_{k}+V_{\mathrm{QD}}(\mathbf{r})\right| 3 / 2,3 / 2\right\rangle$, $E_{\mathrm{hh}}=\left\langle 3 / 2,1 / 2\left|\hat{P}_{k}-\hat{Q}_{k}+V_{\mathrm{QD}}(\mathbf{r})\right| 3 / 2,1 / 2\right\rangle, \quad$ and for the coupling terms, $\left\langle S_{k}\right\rangle=\left\langle 3 / 2,3 / 2\left|\hat{S}_{k}\right| 3 / 2,1 / 2\right\rangle$, $\left\langle R_{k}\right\rangle=\left\langle 3 / 2,3 / 2\left|\hat{R}_{k}\right| 3 / 2,-1 / 2\right\rangle$. The differential operators $\left\{\hat{P}_{k}, \hat{Q}_{k}, \hat{R}_{k}, \hat{S}_{k}\right\}$ will be given in Appendix B. As long as the condition $\Delta_{\mathrm{hh}-\mathrm{lh}}=E_{\mathrm{hh}}-E_{\mathrm{lh}} \gg\left|\left\langle S_{k}\right\rangle\right|,\left|\left\langle R_{k}\right\rangle\right|$ is fulfilled, we have $\beta \sim\left\langle R_{k}\right\rangle / \Delta_{\text {hh-lh }}$ and $\gamma \sim\left\langle S_{k}\right\rangle / \Delta_{\text {hh-lh }}[29]$.

\section{Dependance of the VBM parameters with the geometrical distortions}

Finally, these parameters are evaluated using $\mathrm{HH}$ and $\mathrm{LH}$ Gaussian-like envelope wave functions to which the same transformations used to convert a symmetrical $D_{2 d}$ to the asymmetrical $C_{s} \mathrm{QD}$ potential are applied. The envelope wave functions can then be written as

$\chi_{j}(\mathbf{r})=N^{j} \exp \left[-Z^{2} / 2\left(L_{z}^{j}\right)^{2}-X^{2} / 2\left(L_{x}^{j}\right)^{2}-Y^{2} / 2\left(L_{y}^{j}\right)^{2}\right]$, with $X=x, Y=y \cos \theta-z \sin \theta, Z=z \cos \theta+y \sin \theta$, and $L_{x}^{j}=L_{\|}^{j}(1+\epsilon), L_{y}^{j}=L_{\|}^{j} /(1+\epsilon)$, where $L_{\|}^{j}$ is the effective in-plane confinement length. $N^{j}$ is the normalization factor, and $j \in\{\mathrm{hh}, \mathrm{lh}\}$. The effective confinement length of the $\mathrm{HH}$ and $\mathrm{LH}$ are deduced from their effective-mass difference ratio, $L_{\|}^{\mathrm{lh}}=\kappa_{\|} L_{\|}^{\mathrm{hh}}$ and $L_{z}^{\mathrm{lh}}=\kappa_{z} L_{z}^{\mathrm{hh}}$, with $\kappa_{\|}$and $\kappa_{z}$ being a function of the Luttinger parameters $\gamma_{1}, \gamma_{2}$, and $\gamma_{3}$ (see Appendix B). Using the assumption $L_{\|}^{j} \gg L_{z}^{j}$, we then find

$$
\begin{aligned}
& \beta= 4 \sqrt{6}\left(\frac{L_{z}^{\mathrm{hh}}}{L_{\|}^{\mathrm{hh}}}\right)^{2} \frac{\gamma_{3}}{\gamma_{2}} \frac{\kappa_{\|} \kappa_{z}^{5 / 2}}{\left(1+\kappa_{\|}^{2}\right)^{2}\left(1+\kappa_{z}^{2}\right)^{3 / 2}} \\
& \times {\left[\epsilon+\frac{1}{2}\left(\frac{L_{\|}^{\mathrm{hh}}}{L_{z}^{\mathrm{hh}}}\right)^{2} \frac{\kappa_{\|}^{2}}{\left(1+\kappa_{\|}^{2}\right)\left(1+\kappa_{z}^{2}\right)^{2}} \theta^{2}\right], } \\
& \gamma=-i 2 \sqrt{6} \frac{\gamma_{3}}{\gamma_{2}} \frac{\kappa_{\|} \kappa_{z}^{5 / 2}}{\left(1+\kappa_{\|}^{2}\right)\left(1+\kappa_{z}^{2}\right)^{5 / 2}} \theta .
\end{aligned}
$$

From these equations, we note that (i) $|\gamma|$ is largely independent of the size of the QD, but instead depends on the amplitude of the out-of-plane distortion $\theta$. Since $|\gamma|$ is related to the dark-bright coupling, a similar independence with respect to dot height and a dependence on the out-of-plane distortion $\theta$ is expected for the DB mixing [30], [18]. The relative independence of the DB mixing with the height of the QD has also been shown by more sophisticated atomistic calculations [16]. (ii) $|\beta|$ depends strongly on the aspect ratio of the QD and on the in-plane anisotropy $\epsilon$. However, the out-of-plane distortion $\theta$ also acts to increase the amplitude of $|\beta|$, with a quadratic dependence. This latter point explains the fact that the three dots, among the 25 measured, for which the DE emission was observed in the absence of magnetic field, had higher $\beta$ values (see Fig. 2), i.e., $\beta>0.09$. This is the consequence of the out-of-plane deformation that affects both the dark-bright coupling and the optical anisotropy, i.e., the ellipticity of the polarization diagram along the growth axis.

A quantitative approach for evaluating the VBM parameters is given in Appendix B, where it is shown that an out-of-plane distortion compatible with AFM measurements of the nanohole anisotropy leads to a magnitude of $|\beta|$ close to the mean value experimentally measured (Fig. 2). We will focus next on the direct estimation of magnitude of the darkbright coupling by magneto-PL measurements.

\section{EXPERIMENTS}

\section{A. Dark exciton luminescence}

Figure 4 shows PL and photoluminescence excitation (PLE) spectroscopy of the DE in the absence of applied magnetic field. The working temperature is $\sim 3 \mathrm{~K}$ for all the experimental results. We study the two previously described samples: sample A, where the dots are embedded in $\mathrm{GaAs}$, which is unprocessed, and sample $\mathrm{B}$, where the dots are embedded in an $\mathrm{Al}_{0.33} \mathrm{Ga}_{0.67} \mathrm{As}$ (core) and $\mathrm{Al}_{0.7} \mathrm{Ga}_{0.3} \mathrm{As}$ (cladding) waveguide heterostructure, which has been etched into 1- $\mu \mathrm{m}$-wide one-dimensional (1D) ridges. A monomode fiber, brought close to the cleaved edge, is coupled to the 1D waveguide (WG), while a microscope objective is focused on the top surface (see the inset of Fig. 7). This experimental configuration allows the excitation/detection optical paths to 

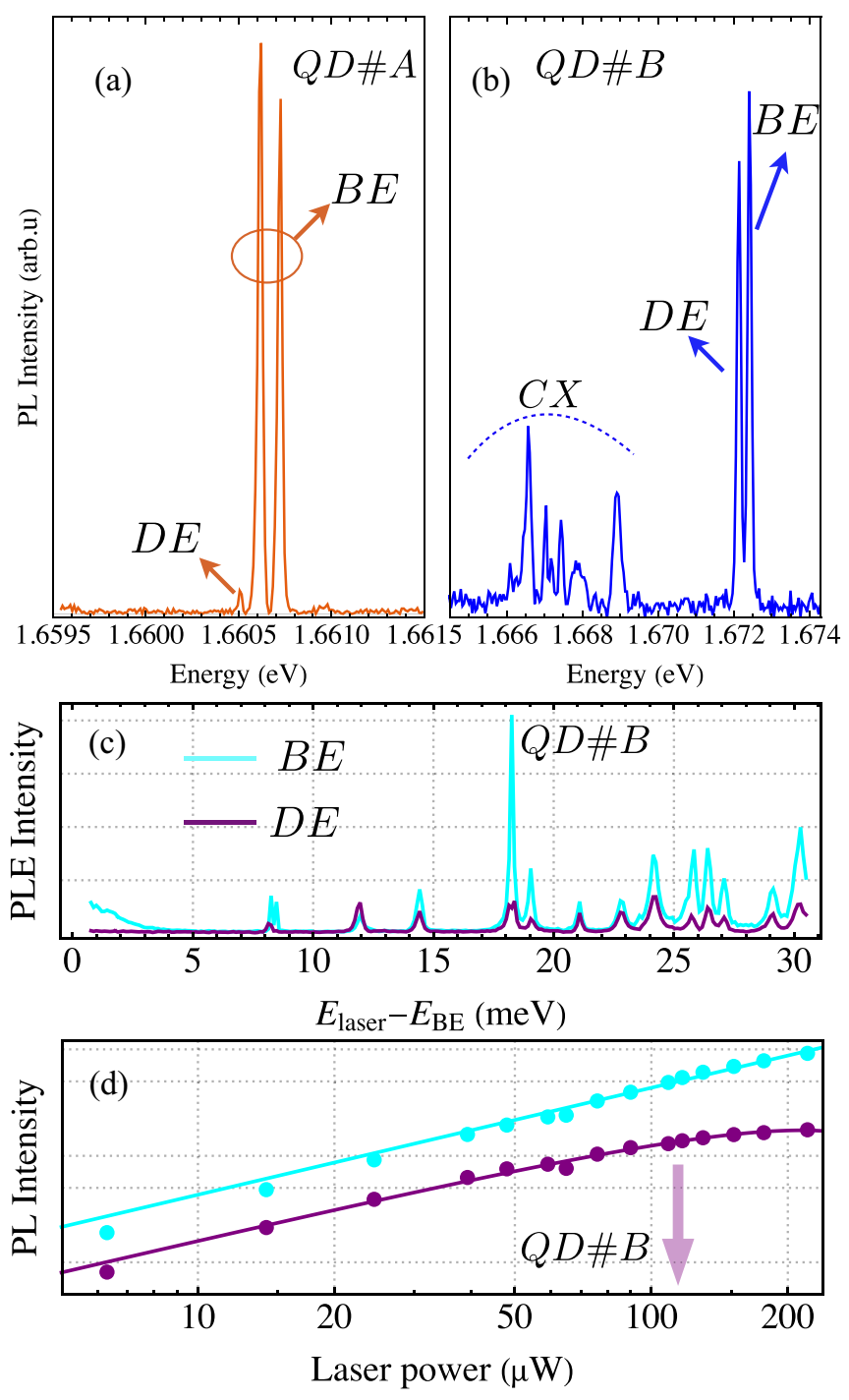

FIG. 4. (a) Typical high-resolution PL spectrum, taken on sample $\mathrm{A}$, showing both the DE and BE signal. A $\lambda / 2$-waveplate was used to balance the intensity of the two BE components. The dot is excited with an above-band-gap excitation using a He-Ne laser. (b) Typical PL spectrum from sample B showing comparable DE and BE intensities for quasiresonant excitation $(\sim 8 \mathrm{meV}$ above the BE energy). Charged excitons (CX) are also observed due to the presence of high $\mathrm{Al}$ concentration in the heterostructure. Note that different optical setups were used for QD\#A and QD\#B. The BE FSS ( $\sim 40 \mu \mathrm{eV}$; see the main text) is not fully resolved for QD\#B, which explains why a single line appears for the two BE components as opposed to QD\#A. (c) PLE spectra of the DE and BE from QD\#B. The laser excites the QD along the 1D waveguide while the PL is collected along the growth axis. A lower laser power has been used than in (b), explaining the different intensity ratio of excitonic resonances at $8 \mathrm{meV}$ than that of PL intensities. As the energy gets closer to the one-LO-phonon resonance, DE lines become less and less pronounced compared to the BE ones. (d) Power-dependent PL of the $\mathrm{DE}$ and $\mathrm{BE}$ resonances located at $8 \mathrm{meV}$. The saturation power for the $\mathrm{DE}$ emission is indicated by an arrow, whereas the $\mathrm{BE}$ emission continues to demonstrate a linear power dependance beyond the DE saturation power.

be switched between edge and growth directions, while still measuring the luminescence from the same QD. The use of a 1D WG allows, on the one hand, to probe QDs located several millimeters away from the cleaved face of the WG in order to avoid any influence of residual strain, and on the other hand, to excite quasiresonantly the QD thanks to an increased spatial rejection of the laser. This $1 \mathrm{D}$ geometry has successfully been used to probe the resonant fluorescence and coherence properties of strained InAs/GaAs QDs [31,32].

Power-dependent PL measurements have been performed, and we restrict ourselves to QDs for which the DE is observable in the absence of an applied magnetic field. When the QD is excited with a He-Ne laser (632.8 nm), the DE PL intensity is very weak. By way of illustration, Fig. 4(a) shows the luminescence intensity of a QD labeled QD\#A on sample $\mathrm{A}$, where the $\mathrm{BE}$ component is $\sim 25$ times stronger than that associated with the DE. The laser power was set at the lowest possible value while still allowing a sufficient signal-to-noise ratio to measure the $\mathrm{DE}$ emission with an acquisition time of $10 \mathrm{~s}$.

In this excitation regime, where the laser energy is much greater than the band gap, and absorption is high, increasing the pump power leads to a fast saturation and eventually extinction of the DE emission. Increasing the luminescence signal of the DE is therefore favored by an excitation regime where the QD captures on average fewer than one $e-h$ pair per radiative cycle of the $\mathrm{DE}$.

As shown in Fig. 4(b), this condition can be fulfilled for an intradot excitation energy resonant with an excitonic excited state (here $8 \mathrm{meV}$ above the BE energy) where the luminescence of the DE becomes comparable to the $\mathrm{BE}$ in the case of sample B. By comparing the PLE spectra of the BE and DE, it can be seen that both share the same excitonic resonances [see Fig. 4(c)]. A detailed study of the excited states is beyond the scope of this paper, however these coincidences reflect the fact that the capture of the dark pair results from an efficient spin relaxation of the carriers from the excited states, i.e., that the same excited state can relax to either a BE or via a spin-relaxation process to a DE. Moreover, the closer the excited state is to the one-LO-phonon resonance energy located at $36 \mathrm{meV}$ above, the more the dark-bright intensity ratio decreases. This indicates a faster optical-phonon assisted relaxation towards the lower-energy states, which promotes the capture of the BE.

Figure 4(d) shows the power dependence of the DE emission when the laser energy is set at $8 \mathrm{meV}$ above the $\mathrm{BE}$, where the DE PL saturation occurs first due to the slower recombination dynamics. We also observe that when the laser is resonant with an excited state of higher energy, the saturation power of the DE decreases (not shown here). This seems to qualitatively confirm the previous arguments: a simple rate equation model shows that the saturation power of the DE, $P_{\text {sat }}$, is proportional to $\left(\tau_{R}+\tau_{S}\right) / \tau_{S}$, where $\tau_{R}$ and $\tau_{S}$ are the effective relaxation time and the spin lifetime of the excited state, respectively. Decreasing $\tau_{R}$ contributes to decreasing $P_{\text {sat }}$.

In the next sections, we describe the measurement of the dark-bright exciton coupling strength leading to the brightening of the DE along the growth direction. Finally, taking advantage of the $1 \mathrm{D}$ waveguide geometry in sample B, a study on the coherence time of the DE state under quasiresonant optical excitation will be presented. 

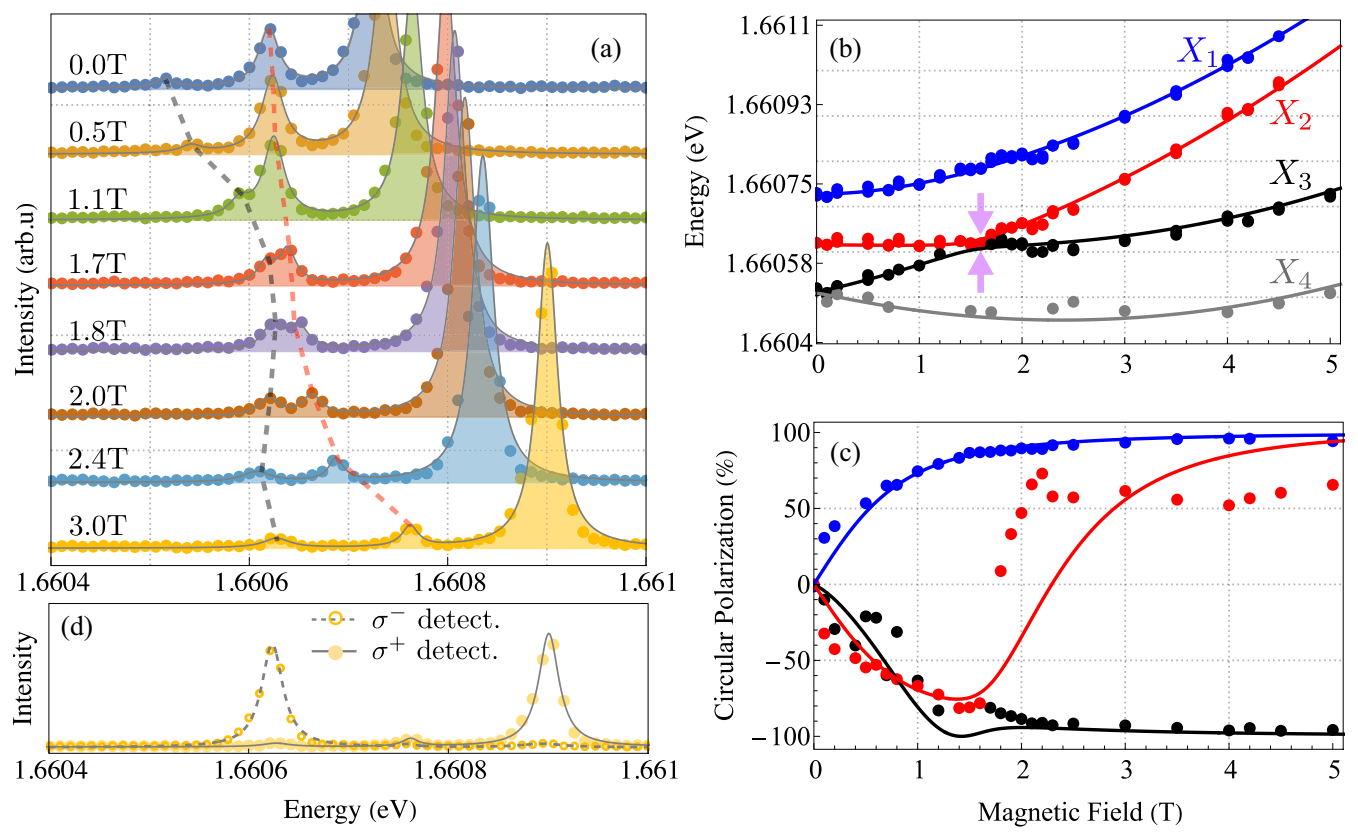

FIG. 5. (a) Luminescence spectra of the QD\#A for different applied magnetic field. The dashed lines are guidelines to highlight the anticrossing signature. The $\lambda / 4$-waveplate is continuously adjusted for each magnetic field so that the nearest bright state does not hide the DE signal. When the magnetic field exceeds the anticrossing, the $\lambda / 4$-waveplate position corresponds to a $\sigma^{+}$analysis, minimizing the intensity of the $X_{3}$ branch while maximizing that of $X_{1}$. (b) Zeeman spectroscopy of the four excitonic lines from QD\#A, which shows a level anticrossing at $1.8 \mathrm{~T}$ between branches $X_{2}$ and $X_{3}$. The diamagnetic shift of $10.76 \pm 0.08 \mu \mathrm{eV} \mathrm{T}^{-2}$ is deduced from the fitting procedure. (c) Degree of circular polarization as a function of the magnetic field. The lines are fits using the values from Table I. (d) $\sigma^{+} / \sigma^{-}$polarization-resolved PL spectra for a magnetic field of $3 \mathrm{~T}$.

\section{B. Magneto-PL experiments}

To measure the dark-bright exciton coupling [33,34], a longitudinal magnetic field was applied to QD\#A. The experimental conditions are the same as those used in Fig. 4(a). We used here a Jobin-Yvon U1000 dual spectrometer with a $10 \mu \mathrm{eV}$ resolution. Figure 5(a) shows the luminescence spectra for different applied magnetic fields, tracking the evolution of the dark and bright states. The emission lines are associated with the dark exciton, and the bright doublet at higher energy is clearly identified, as in Fig. 4(a). The angle of the $\lambda / 4$ waveplate placed on the detection path is adjusted for each magnetic field to minimize the intensity of the bright state closest to the DE, allowing us to follow precisely its spectral evolution. Figure 5(d) shows the sigma+/sigmaresolved spectra at $3 \mathrm{~T}$, where all peaks start to exhibit a high degree of circular polarization. This gives an estimation of the PL intensity of each line in the strong magnetic field region, complementing the intensities measured at zero field in Fig. 4(a). Figure 5(b) shows the energy dependence of the $\mathrm{BE}$ and DE as a function of the magnetic field amplitude. We observed four branches evolving as expected.

For clarity, we labeled them as branches $X_{1}-X_{4}$, in decreasing order of their energy position at zero field. Branches $X_{1}, X_{2}$ correspond to the bright exciton, and branches $X_{3}, X_{4}$ correspond to the dark exciton at $0 \mathrm{~T}$. The striking feature is the presence of a weak anticrossing (AC) between the intermediate branches $X_{2}$ and $X_{3}$ at $B_{\mathrm{AC}}=1.8 \mathrm{~T}$. This anticrossing reflects the existence of the coupling between $\mathrm{BE}$ and $\mathrm{DE}$, and it explains a posteriori the brightening of the DE in the absence of a magnetic field.

The anticrossing amplitude of $15 \mu \mathrm{eV}$ is well reproduced by the diagonalization of the total Hamiltonian $\hat{H}=\hat{H}_{\text {exc }}+$ $\delta \hat{H}_{C_{s}}+\hat{H}_{z}$, where the $e-h$ exchange Hamiltonian is written as

$$
\begin{aligned}
\hat{H}_{\mathrm{exc}}= & \frac{\Delta_{0}}{2} \sum_{\substack{i \in\{+1,-1\} \\
j \in\{+2,-2\}}}[|i\rangle\langle i|-| j\rangle\langle j|] \\
& +\frac{\Delta_{1}}{2}|+1\rangle\left\langle-1\left|+\frac{\Delta_{2}}{2}\right|+2\right\rangle\langle-2|+\text { H.c., }
\end{aligned}
$$

and the Zeeman Hamiltonian is

$$
\begin{aligned}
\hat{H}_{z}= & \frac{g_{B}^{z} \mu_{B} B_{z}}{2}(|+1\rangle\langle+1|-|-1\rangle\langle-1|) \\
& +\frac{g_{D}^{z} \mu_{B} B_{z}}{2}(|+2\rangle\langle+2|-|-2\rangle\langle-2|) .
\end{aligned}
$$

$\Delta_{0}$ is the amplitude of the isotropic $e-h$ exchange interaction, $\Delta_{1}\left(\Delta_{2}\right)$ corresponds to the isotropic component acting on the BE (DE), and $g_{B}^{z}\left(g_{D}^{z}\right)$ is the effective BE (DE) exciton longitudinal Landé $g$-factor. Note that because of $\delta \hat{H}_{C_{s}}$, the dark-bright splitting $\Delta_{\mathrm{DB}}$ is no longer $\Delta_{0}$, and for the same reason $\Delta_{1}\left(\Delta_{2}\right)$ is no longer the fine-structure splitting of the BE (DE) $[17,18]$. Table I lists the parameters obtained from the best fit to the data shown in Fig. 5(b).

The degree of circular polarization, $\rho_{c}$, defined as $\left(I^{\sigma^{+}}-\right.$ $\left.I^{\sigma^{-}}\right) /\left(I^{\sigma^{+}}+I^{\sigma^{-}}\right)$, is a good observable, sensitive to the dark- 
bright exciton coupling, as its evolution with the applied magnetic field confirms the presence of the anticrossing. The nonresonant excitation ensures the equal distribution of the exciton populations. As a consequence, $\rho_{c}$ probes the weight of the bright components $|+1\rangle$ and $|-1\rangle$. Figure 5(c) maps the evolution of the circular polarization of the $X_{1}-X_{3}$ branches as a function of applied magnetic field. The most striking feature is the nonmonotonic behavior of the $X_{2}$ circular polarization and its sign inversion for a magnetic field amplitude greater than $B_{\mathrm{AC}}$.

When the magnetic field is weak, the branch $X_{2}$ shows a negative degree of circular polarization that increases at the same rate as $X_{1}$ but with opposite sign as the magnetic field increases. In this field regime, the bright component of the DE develops a character, since the branch $X_{3}$ shows a negative circular polarization when getting closer to the branch $X_{2}$ with a maximum at the anticrossing position where the energy separation is minimal. Far from the anticrossing, the DE character is progressively transferred to the branch $X_{2}$. This is demonstrated by the circular polarization of the different branches shown in Fig. 5(c). $X_{2}$ develops a positive circular polarization, similar to $X_{1}$, due to the dark-bright coupling between the $X_{2}$ and $X_{1}$ branches as they approach in energy for $B_{z}>B_{\mathrm{AC}}$. The $X_{2}$ emission is much less intense than $X_{1}$ under measurement conditions that optimize collection of polarized light [Fig. 5(d)]. In contrast, branch $X_{3}$ has the opposite circular polarization to $X_{1}$ at high magnetic field, and it corresponds to the oppositely polarized bright state [Fig. 5(d)].

In this qualitative demonstration, we have considered implicitly that the couplings of the $\mathrm{DE}$ to the $\mathrm{BE}|+1\rangle$ and $|-1\rangle$ are of the same order of magnitude. Therefore, only the proximity in energy to the $|+1\rangle$ or $|-1\rangle$ states governs the sign of its circular polarization. This allows us to understand the sign inversion of the circular polarization for the branch $X_{2}$ as $\left|\Delta_{11}\right| \sim\left|\Delta_{12}\right|$ (see Table I). This last condition is also consistent with the high degree of linear polarization of the dark exciton measured at zero field as shown hereafter. Appendix C provides the asymptotic dependence of $\rho_{c}$ on each side of the anticrossing demonstrating the change of sign.

The solid curves in Fig. 5(c) are numerical calculations using the parameters given in Table I. Note that for each value of the applied magnetic field, the finite value of $\beta$ has been taken into account for the numerical estimation of $\rho_{c}$. In particular, the limit of the circular polarization in a strong magnetic field

TABLE I. Parameters obtained from analysis of the linear polarization diagram of QD A, and from the best fit to the Zeeman spectroscopy of the four exciton lines shown in Fig. 5(b).

\begin{tabular}{lc}
\hline \hline Parameters & Best-fit value \\
\hline$\Delta_{0}$ & $149.0 \pm 3 \mu \mathrm{eV}$ \\
$\Delta_{1}$ & $102.0 \pm 3 \mu \mathrm{eV}$ \\
$\Delta_{2}$ & $-9.0 \pm 5 \mu \mathrm{eV}$ \\
$\Delta_{11}$ & $-48.0 \pm 5 \mu \mathrm{eV}$ \\
$\Delta_{12}$ & $36.0 \pm 6 \mu \mathrm{eV}$ \\
$g_{B}^{z}$ & $1.36 \pm 0.03$ \\
$g_{D}^{z}$ & $1.79 \pm 0.03$ \\
$|\beta|$ & 0.16 \\
\hline \hline
\end{tabular}
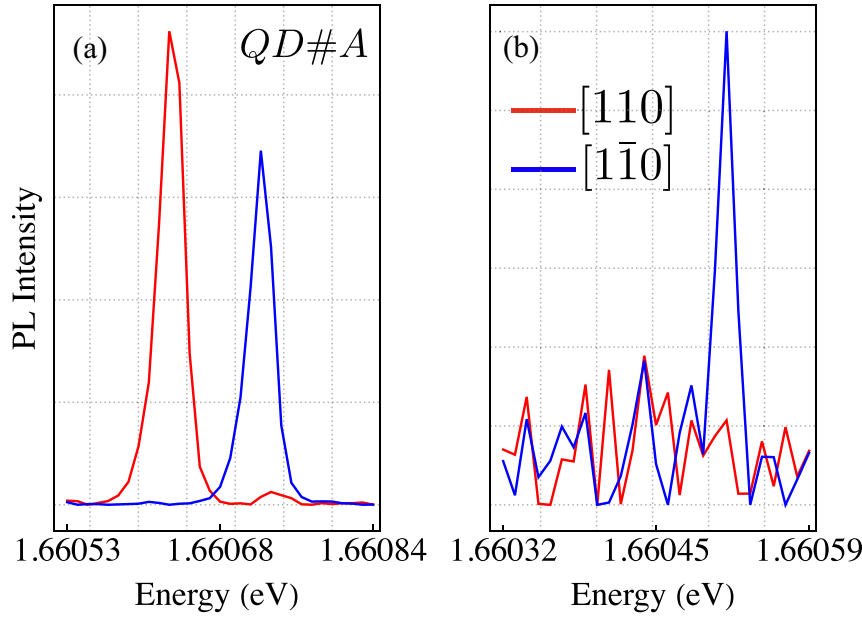

FIG. 6. Linearly polarized resolved spectra of the BE (a) and DE (b) from QD\#A, showing a degree of linear polarization close to $100 \%$ for the DE, and of the same sign as the high-energy component of the BE. Note that QD\#B (not shown here) shares the same features.

tends to $\pm\left(1-|\beta|^{2} / 3\right) /\left(1+|\beta|^{2} / 3\right)$ [35]. The calculations reproduce well the trend of the circular polarization evolution. Nevertheless, experimentally the change in sign of the $X_{2}$ branch circular polarization shows a more rapid transition than that described by the calculations as it passes through the anticrossing regime. It is possible that a residual helicity of the laser creates a weak nuclear polarization, too weak to be measured on the splittings, but which could modify the circular polarization [36]. This effect is all the more sensitive when the circular polarization is weak.

Figure 6 shows linearly polarized spectra of the $\mathrm{BE}$ and $\mathrm{DE}$ in the absence of applied magnetic field. It can be seen that the DE is close to $100 \%$ polarized and has the same polarization as the higher-energy component of the BE. This can be explained by considering the emission of the DE in the absence of magnetic field, taking into account that $\left|\Delta_{11}\right|$ and $\left|\Delta_{12}\right|$ are very close in magnitude (see Table I). We can thus introduce an effective dark-bright exciton coupling term $\widetilde{\Delta} \sim \Delta_{12} \sim-\Delta_{11}$ with $\widetilde{\Delta}=\left(\left|\Delta_{12}\right|+\left|\Delta_{11}\right|\right) / 2$ to simplify the description of the DE emission. The DE states in the absence of magnetic field, labeled $\mathrm{DE}_{H}$ and $\mathrm{DE}_{L}$, can now be written [6] as

$$
\begin{aligned}
&\left|\mathrm{DE}_{H}\right\rangle=N[|+2\rangle-|-2\rangle \\
&\left.+\frac{\widetilde{\Delta}}{\Delta_{0}+\Delta_{1} / 2}(|+1\rangle+|-1\rangle)\right], \\
&\left|\mathrm{DE}_{L}\right\rangle=[|+2\rangle+|-2\rangle] / \sqrt{2},
\end{aligned}
$$

where $N$ is the normalization factor. The index $H(L)$ refers to the high-energy (low-energy) side.

These expressions show that the $\mathrm{DE}_{H}$ is antisymmetric (AS) and the $\mathrm{DE}_{L}$ is symmetric (S) when considering only the $|+2\rangle,|-2\rangle$ components. The (S) DE has no bright component [37], explaining why it is not observable at zero magnetic 


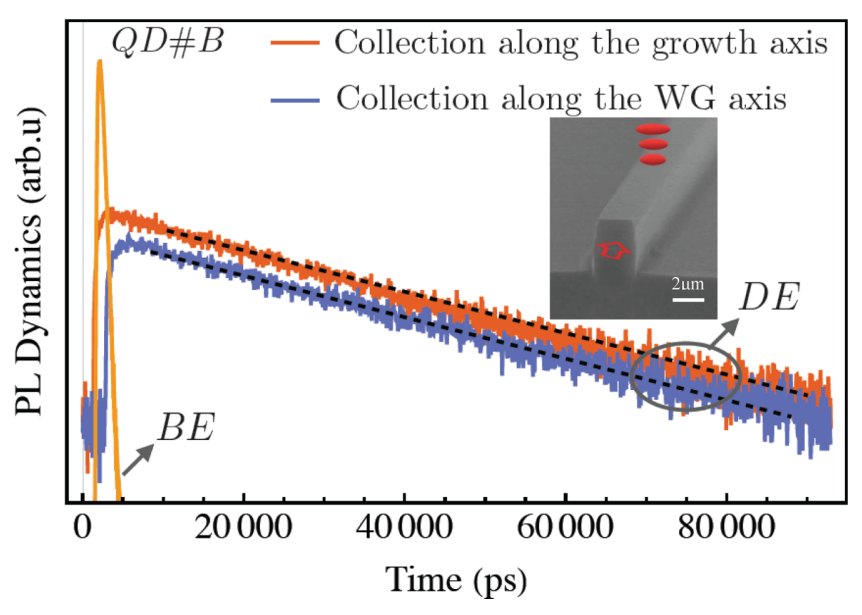

FIG. 7. Time-resolved PL of the DE recorded either along the growth axis or along the direction of the waveguide, giving the same decay time of $46 \mathrm{~ns}$. For comparison, the time-resolved PL of the BE is also represented on the same log-log plot, showing a decay time of $0.46 \mathrm{~ns}$. The inset shows a SEM image of a ridge and the orthogonal excitation/detection geometry that is used.

field and is even very difficult to be detected when increasing the magnetic field. This is the reason why the branch $X_{4}$ is plotted with only a few experimental points in Fig. 5(b). In contrast, the AS DE has a symmetric linear combination of the $|+1\rangle$ and $|-1\rangle$ states. This indicates that the AS DE emission will have the same polarization as the $\mathrm{BE}_{H}$, explaining the polarization-resolved PL shown in Figs. 6(a) and 6(b). This correlation in polarization is valid when the conditions $\Delta_{2}<0$ and $\tilde{\Delta}<\sqrt{1 / 2\left(\Delta_{2}^{2}-2 \Delta_{2} \Delta_{0}-\Delta_{2} \Delta_{1}\right)}$ are fulfilled, which is the case here. We note that for InGaAs QDs, the linear polarization of the DE was observed to be the same as that of the lower-energy bright exciton $\mathrm{BE}_{L}$ [2] as a consequence of different values of the $e-h$ exchange energies.

\section{Coherence properties of the dark exciton state}

The DE of QD\#B shows the same high degree of linear polarization, indicating that $\left|\Delta_{12}\right| \sim\left|\Delta_{11}\right|$. This high degree of linear polarization can be used to selectively increase the DE emission in order to study its dynamical properties.

Figure 7 shows time-resolved PL spectra for the DE and $\mathrm{BE}$ of $\mathrm{QD \# B}$. A picosecond laser, tuned at about $60 \mathrm{meV}$ above the energy of the $\mathrm{BE}$, excites the QD with a repetition rate of $\sim 11 \mathrm{MHz}$ in order to measure very slow dynamics. The PL is dispersed through a 0.5 -m-focal-length spectrometer coupled to a fast avalanche photodiode (APD) equipped with a photon-counting module, giving a time resolution of $\sim 200$ ps. The excitation energy was selected to minimize the scattered light on the APD without degrading significantly the DE signal intensity.

The dynamics of the PL is analyzed on the same dot using two experimental configurations: (i) exciting perpendicular to the top surface and collecting the PL emitted along the waveguide, and (ii) exciting via the $1 \mathrm{D}-\mathrm{WG}$ and collecting the light along the growth axis.

Although the emission of the DE has a different origin depending on whether the luminescence is collected perpen- dicular or parallel to the growth axis, the same decay time $\tau_{\mathrm{rad}}^{\mathrm{DE}}=46 \mathrm{~ns}$ has been measured. This reflects the fact that the PL signal is proportional to the DE population, even though the DE is coupled differently to the parallel and perpendicular electromagnetic field modes. As a comparison, the PL dynamics of the BE is also represented on the same graph, and gives a radiative decay time $\tau_{\text {rad }}^{\mathrm{BE}} \sim 500$ ps.

The absence of biexponential behavior on both $\mathrm{BE}$ and $\mathrm{DE}$ decays, and more particularly on the $\mathrm{BE}$, indicates that the temperature $(\sim 3 \mathrm{~K})$ is too low to convert the DE population into a reservoir for the $\mathrm{BE}$ through acoustic phonon scattering [38]. As a consequence, the DE decay is predominantly radiative [2]. This is also confirmed by the fact that from Eq. (10), we can approximate the ratio $\tau_{\mathrm{rad}}^{\mathrm{DE}} / \tau_{\mathrm{rad}}^{\mathrm{BE}}$ as $\left(\frac{\tilde{\Delta} / 2}{\Delta_{0}+\Delta_{1} / 2}\right)^{2}$, giving $\tilde{\Delta} \sim 50 \mu \mathrm{eV}$, which agrees well with the values found in this type of QD. For this estimation, we used the approximations $\Delta_{\mathrm{DB}} \sim \Delta_{0}$ and the BE FSS closed to $\sim \Delta_{1}$, with $\Delta_{\mathrm{DB}}(240 \mu \mathrm{eV})$ and BE FSS $(40 \mu \mathrm{eV})$ being experimentally measured. As the correction is equal to $\tilde{\Delta}^{2} / \Delta_{0} \sim 1 \mu \mathrm{eV}$, the approximation used here is valid $[17,18]$.

Figures 8(a) and 8(b) show the interference contrast for the $\mathrm{BE}$ and $\mathrm{DE}$ of $\mathrm{QD \# B}$ as a function of the time delay between the two arms of the Michelson interferometer [39]. For these experiments, the QD is excited with a CW laser tuned at $8 \mathrm{meV}$ above the bright excitonic transition. The interferogram patterns are Gaussian-shaped, and we define $T_{\Sigma}$ as the full width at half-maximum of the contrast. We find for the BE a $T_{\Sigma}^{\mathrm{BE}}$ of $30 \mathrm{ps}$ and for the DE a $T_{\Sigma}^{\mathrm{DE}}$ of $48 \mathrm{ps}$. These times are extremely short compared to the radiative lifetime, and the Gaussian shape points towards an efficient spectral diffusion mechanism leading to an inhomogeneous broadening of the QD [40-42]. The charge noise can be attributed to the high aluminum content of the AlGaAs waveguide and the impurities in the aluminum source during the sample growth.

The data in Figs. 8(a) and 8(b) show that $T_{\Sigma}^{\mathrm{DE}}$ is $33 \%$ longer than $T_{\Sigma}^{\mathrm{BE}}$, and Fig. 8(c) shows that $T_{\Sigma}^{\mathrm{DE}}>T_{\Sigma}^{\mathrm{BE}}$ regardless of the excitation power. Similar results are found when using the other experimental configuration, i.e., when collecting the PL signal along the waveguide. Although the excitation power required in this orientation was much higher, the same experimental trends were found with $T_{\Sigma}^{\mathrm{DE}}=30 \mathrm{ps}$ and $T_{\Sigma}^{\mathrm{BE}}=20 \mathrm{ps}$, confirming the electrostatic noise as the dominant mechanism in this sample. To understand better why the coherence properties of the DE are less sensitive to the impact of the electrostatic fluctuations than those of its bright counterpart, we present a simple model that is qualitatively consistent with the experimental results. The fluctuating electrostatic field is defined by the moments of its distribution, i.e., $\langle\delta F(t)\rangle=0,\left\langle\delta F^{2}(t)\right\rangle=\sigma_{F}^{2}$, and its autocorrelation function $\langle\delta F(\tau) \delta F(0)\rangle=\sigma_{F}^{2} \exp \left(-|\tau| / \tau_{c}\right)$, with $\tau_{c}$ the correlation time [43]. Assuming that the normal distribution describes the charge reservoir, the spectral-diffusion-induced interference contrast $C(t)$ is given by

$$
C(t)=\exp \left[-\frac{\Sigma^{2} t^{2}}{\hbar^{2}}\left(\exp \left(-t / \tau_{c}\right)+t / \tau_{c}-1\right)\right],
$$

where $\Sigma=\sqrt{\left\langle\delta E(t)^{2}\right\rangle}$, and $\delta E(t)$ is the energy fluctuation around the average value, i.e., $\langle\delta E(t)\rangle=0$. When the 

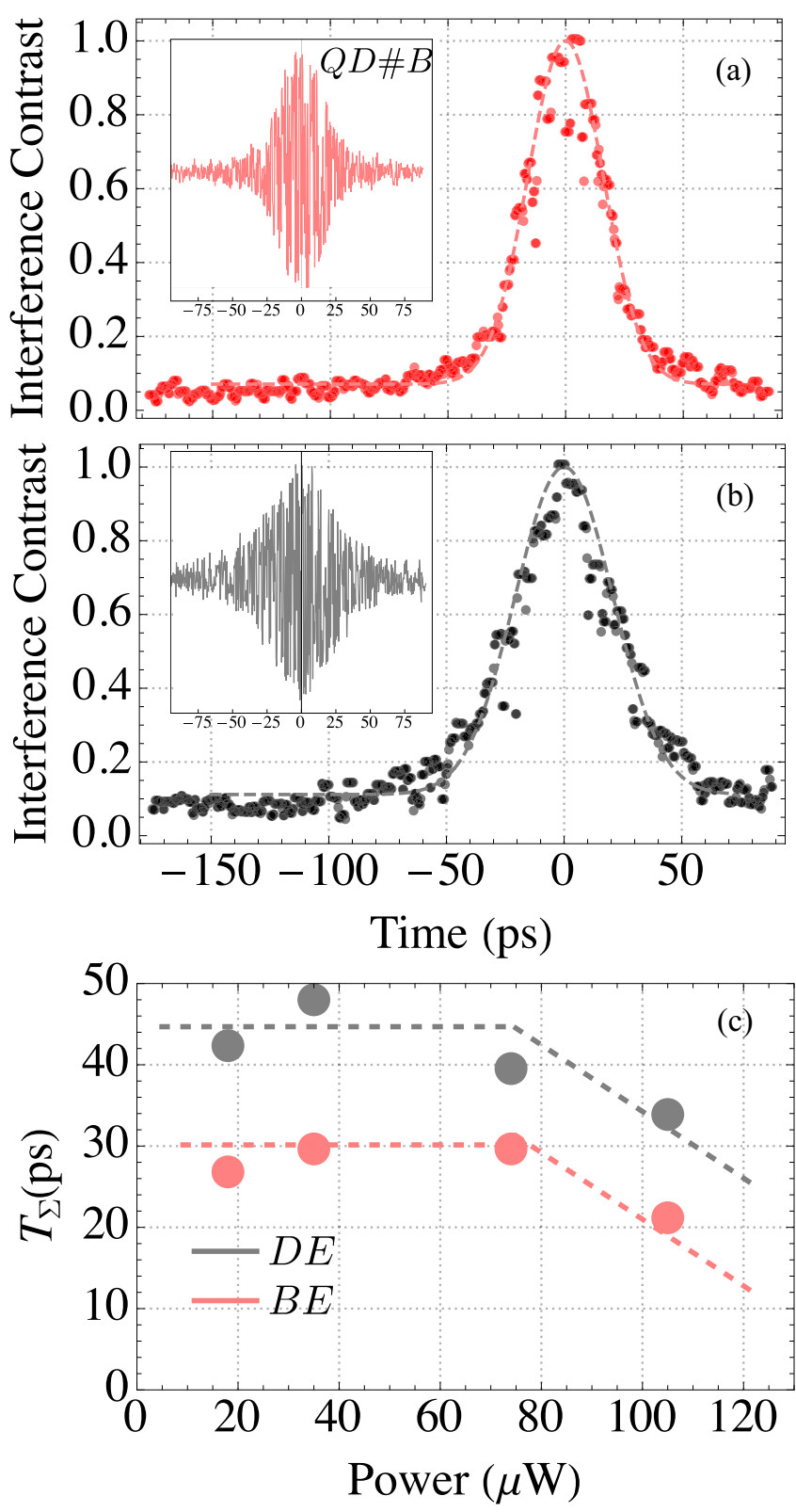

FIG. 8. Interferogram contrast as a function of the path delay time between the two arms of the interferometer for the BE (a) and the DE (b). The insets show the raw interferograms. The laser power is set at $38 \mu \mathrm{W}$, and the laser energy is $8 \mathrm{meV}$ above the BE. (c) Evolution of $T_{\Sigma}$ as a function of the laser power for both DE and BE.

condition $t \leqslant T_{\Sigma} \ll \tau_{c} \sim 1 \mu \mathrm{s}$ is satisfied, $C(t)$ can be well approximated by $C(t) \sim \exp \left[-\frac{\Sigma^{2} t^{2}}{2 \hbar^{2}}\right]$, and $T_{\Sigma}=2 \sqrt{\ln 2} \hbar / \Sigma$.

To estimate the ratio $T_{\Sigma}^{\mathrm{DE}} / T_{\Sigma}^{\mathrm{BE}}=\Sigma^{\mathrm{BE}} / \Sigma^{\mathrm{DE}}$, we present a simple model where the spectral diffusion is induced by a confined Stark effect having two contributions. The first one is carried by the interaction of the static dipole moment $\mu_{0}$ with the electric field supposed to be the same for the DE and BE [44]. The second one takes into account the influence of the electric field on the amplitude of the fine structure of the
BE, $\Delta_{1}(t)$, and the DE, $\Delta_{2}(t)$, which can be written, to lowest order in the VBM parameters, as [45]

$$
\begin{aligned}
& \Delta_{1}(t)=\Delta_{1}^{0}+\frac{4}{\sqrt{3}} \beta(t) \Delta_{\mathrm{DB}}, \\
& \Delta_{2}(t)=\Delta_{2}^{0}+\frac{4}{3} \beta^{2}(t) \Delta_{\mathrm{DB}},
\end{aligned}
$$

where $\Delta_{1}^{0}\left(\Delta_{2}^{0}\right)$ is the electrostatic-field independent part of the BE FSS (DE FSS). $\Delta_{\mathrm{DB}} \sim \Delta_{0}$ if the condition $\tilde{\Delta} \ll \Delta_{0}$ is fulfilled. This dependence between the FSS, the exchange parameters, and $\beta$ is provided by the short-range $e-h$ exchange interaction [17]. Finally, the effect of the electrostatic field can be described in terms of fluctuations of the VBM parameter as $\beta(t)=-a \delta F(t)+\langle\beta\rangle$, with $a=d \beta / d F$, which has been evaluated at $2.1 \times 10^{-3} \mathrm{kV}^{-1} \mathrm{~cm}$ in the case of InAs/GaAs QD by atomistic calculations [45], and it represents the tunability of the excitonic FFS with the applied electric field, leading to the BE FSS cancellation. As the magnitude of $a$ is very weak, we use the assumption $\mu_{0} \ll a \Delta_{\mathrm{DB}}$, which gives for the $\mathrm{DE}$ and the $\mathrm{BE}$

$$
\begin{gathered}
\delta E^{\mathrm{DE}}(t) \sim-\mu_{0} \delta F(t)-\frac{2}{3} a \Delta_{\mathrm{DB}}\langle\beta\rangle \delta F(t), \\
\delta E^{\mathrm{BE}}(t)=-\mu_{0} \delta F(t)-\frac{2}{\sqrt{3}} a \Delta_{\mathrm{DB}} \delta F(t) .
\end{gathered}
$$

We find $T_{\Sigma}^{\mathrm{DE}} / T_{\Sigma}^{\mathrm{BE}} \sim 1+\frac{2}{3} \frac{a}{\mu_{0}} \Delta_{\mathrm{DB}}(\sqrt{3}-2\langle\beta\rangle)$, which is greater than unity, in agreement with the experiment. Considering very reasonable values of $\langle\beta\rangle=0.15$ and $\mu_{0}=|e| \times$ $0.2 \mathrm{~nm}$, an estimation of $a$ gives $5.7 \times 10^{-2} \mathrm{kV}^{-1} \mathrm{~cm}$, which is an order of magnitude larger than in InAs QDs [45]. Qualitatively, this result seems appropriate since the electrical tuning of the excitonic FSS measured experimentally operates for weaker electric field in GaAs QDs [46] than in InAs QDs [47]. Although this simple model is probably not able to describe correctly the asymptotic behavior of the $T_{\Sigma}^{\mathrm{DE}} / T_{\Sigma}^{\mathrm{BE}}$ ratio when $\langle\beta\rangle$ tends to zero, the study of this ratio for finite values of $\langle\beta\rangle$ is interesting. We note that $T_{\Sigma}^{\mathrm{DE}} / T_{\Sigma}^{\mathrm{BE}}$ is a decreasing function of $\langle\beta\rangle$. This indicates that the mechanism giving rise to DE brightening can also act as a source of coherence loss for the DE itself. In the above model, the DE emission perpendicular to the growth axis was described in terms of the parameter $\beta$. Since the parameter $\gamma$ only appears in higherorder terms in the expansion of the DE FSS in Eq. (18), it was neglected here, especially since its dependence with the electric field is still an open question.

However, this model describes how the coherence loss induced by the fluctuating electrostatic environment can play a weaker role in the coherence properties of the DE, in accordance with the experimental results.

\section{CONCLUSIONS}

The emission properties of the DE confined in strain-free $\mathrm{GaAs} / \mathrm{AlGaAs}$ QDs have been characterized. Brightening of the DE in the absence of applied magnetic field has been seen for around $10 \%$ of dots measured. This has been explained by the fact that these dots, fabricated by partial infilling of an asymmetric nanohole, can have a strong out-of-plane dot asymmetry. The dark-bright coupling at the origin of 
the luminescence along the growth axis has been measured by magneto-optical experiments. The sign inversion of the circular polarization associated with the DE branch when increasing the longitudinal magnetic field above the anticrossing region confirms the presence of this coupling.

We have also shown qualitatively that an intradot excitation at low excitation power enhances the luminescence of DE, whose decay time is radiatively limited since the nonradiative processes seem to play no role. Under quasiresonant excitation, the coherence properties of the dark and bright exciton of the same QD have been measured simultaneously, showing that, although short, the width of the DE interferogram is $33 \%$ longer than that of its bright counterpart. A simple model was presented to account for this experimental result, showing that the DE coherence is more robust and less sensitive to a fluctuating electrostatic environment.

This result should be further investigated by additional experiments in field-effect devices allowing a control of the electrostatic environment [48] and by theoretical studies in order to better understand the subtle features of dark excitons.

\section{ACKNOWLEDGMENTS}

This work was funded by the French National Research Agency (ANR "SPINEX" ANR-17-CE30-0022-01 and ANR "ISQUAD" ANR-18-CE47-0006-01), by the Paris Île-deFrance Région in the framework of DIM SIRTEQ, and by the Cluster of Excellence MATISSE led by Sorbonne Universités. We thank L. Thevenard and M. Vabre for technical support.

\section{APPENDIX A: BLOCH WAVE FUNCTIONS}

The Bloch wave functions can be written as

$$
\begin{aligned}
\left|u_{3 / 2}\right\rangle & =\frac{1}{\sqrt{2}}|X+i Y, \uparrow\rangle, \\
\left|u_{1 / 2}\right\rangle & =\frac{1}{\sqrt{6}}|X+i Y, \downarrow\rangle-\sqrt{\frac{2}{3}}|Z, \uparrow\rangle, \\
\left|u_{-1 / 2}\right\rangle & =-\frac{1}{\sqrt{6}}|X-i Y, \uparrow\rangle-\sqrt{\frac{2}{3}}|Z, \downarrow\rangle, \\
\left|u_{-3 / 2}\right\rangle & =-\frac{1}{\sqrt{2}}|X-i Y, \downarrow\rangle,
\end{aligned}
$$

where $|X\rangle,|Y\rangle$, and $|Z\rangle$ transform like the atomic orbitals $p_{x}$, $p_{y}$, and $p_{z}$ when one applies to them the transformations leaving invariant the local tetrahedron of the zinc-blende crystal, and $\uparrow, \downarrow$ are the valence electron spin components quantified along the $z$ axis.

In the frameworks of the envelope wave-function approximation, the $\mathrm{HH}$ wave functions are $\langle\mathbf{r} \mid 3 / 2, \pm 3 / 2\rangle=$ $\chi_{\mathrm{hh}}(\mathbf{r}) u_{ \pm 3 / 2}(\mathbf{r})$, and those of the LH are $\langle\mathbf{r} \mid \pm 3 / 2,1 / 2\rangle=$ $\chi_{\mathrm{lh}}(\mathbf{r}) u_{ \pm 1 / 2}(\mathbf{r})$.

Taking into account the different mass tensor between $\mathrm{HH}$ and LH leads to different confinement lengths. For a flat QD with the growth direction along $z$, we use $L_{i j}^{\mathrm{lh}}=\overline{\bar{\kappa}}_{i k} L_{k j}^{\mathrm{hh}}$ with $\overline{\bar{\kappa}}=\operatorname{diag}\left[\kappa_{\|}, \kappa_{\|}, \kappa_{z}\right], \kappa_{\|}=\left(\frac{\gamma_{1}-\gamma_{2}}{\gamma_{1}+\gamma_{2}}\right)^{1 / 4}$, and $\kappa_{z}=\left(\frac{\gamma_{1}+2 \gamma_{2}}{\gamma_{1}-2 \gamma_{2}}\right)^{1 / 4}$.

\section{APPENDIX B: NUMERICAL ESTIMATION OF THE VBM PARAMETERS}

To calculate the VBM parameters $\beta$ and $\gamma$, which are accessible from optical measurements [27] as a function of the QD shape, one needs first to evaluate the hole envelope wave functions.

The single-hole particle wave function is calculated in the framework of the four-band Luttinger $\mathbf{k} \cdot \mathbf{p}$ model. The hole states $\mid \widetilde{\left.\psi^{i}\right\rangle}$ with $i \in\{3 / 2,1 / 2,-1 / 2,-3 / 2\}$ are expressed in the basis of the $\Gamma$-point Bloch wave functions as a 4 -vector $\left(\phi_{3 / 2}^{i}(\mathbf{r}), \phi_{1 / 2}^{i}(\mathbf{r}), \phi_{-1 / 2}^{i}(\mathbf{r}), \phi_{-3 / 2}^{i}(\mathbf{r})\right)$ solution of the eigensystem $\hat{H}_{h}=\hat{H}_{L}+V_{\mathrm{QD}(\epsilon, \theta)}^{\mathrm{VB}}(\mathbf{r}) I_{4 \times 4}$ with

$$
\hat{H}_{L}=\left(\begin{array}{cccc}
\hat{P}_{k}+\hat{Q}_{k} & -\hat{S}_{k} & \hat{R}_{k} & 0 \\
-\hat{S}_{k}^{\star} & \hat{P}_{k}-\hat{Q}_{k} & 0 & \hat{R}_{k} \\
R_{k}^{\star} & 0 & \hat{P}_{k}-\hat{Q}_{k} & \hat{S}_{k} \\
0 & \hat{R}_{k}^{\star} & \hat{S}_{k}^{\star} & \hat{P}_{k}+\hat{Q}_{k}
\end{array}\right) \text {, }
$$

where $\hat{P}_{k}=-\frac{\hbar^{2} \gamma_{1}}{2 m_{0}} \nabla^{2}, \hat{Q}_{k}=\frac{\hbar^{2} \gamma_{2}}{2 m_{0}}\left(-\nabla^{2}+3 \frac{\partial^{2}}{\partial z^{2}}\right), \hat{R}_{k}=\frac{\hbar^{2}}{2 m_{0}}(-$ $\left.\sqrt{3} \gamma_{3}\left(\frac{\partial^{2}}{\partial y^{2}}-\frac{\partial^{2}}{\partial x^{2}}\right)-2 i \sqrt{3} \gamma_{2} \frac{\partial}{\partial x} \frac{\partial}{\partial y}\right)$, and $\hat{S}_{k}=\frac{\hbar^{2} \gamma_{3}}{2 m_{0}}\left(i \sqrt{3}\left(i \frac{\partial}{\partial x}+\right.\right.$ $\left.\frac{\partial}{\partial y}\right) \frac{\partial}{\partial z}$ ). The Luttinger parameters $\gamma_{1}=6.85, \gamma_{2}=2.1$, and $\gamma_{3}=2.9$ are extracted from the literature [49].

The valence-band potential is defined as

$$
V_{\mathrm{QD}(\epsilon, \theta)}^{\mathrm{VB}}(\mathbf{r})= \begin{cases}0 & \text { if } \mathbf{r} \in V(\epsilon, \theta), \\ V_{\mathrm{off}}^{\mathrm{VB}} & \text { otherwise, }\end{cases}
$$

with the band offset $V_{\text {off }}^{\mathrm{VB}}=195 \mathrm{meV}$, calculated for $\mathrm{Al}_{0.36} \mathrm{Ga}_{0.64} \mathrm{As}$ in the QD barriers, and $V(\epsilon, \theta)$ is the geometric region defining the inside of the $\mathrm{QD}$ according to Fig. 3. The four-band $\mathbf{k} \cdot \mathbf{p}$ model is implemented by a finite-element method using a discretization volume larger than the volume of $V_{\mathrm{QD}(\epsilon, \theta)}(\mathbf{r})$ to immerse the QD in the AlGaAs barrier material. The discretization volume contains typically $9.0 \times 10^{6}$ voxels and is adapted as a function of the QD size in order to minimize boundary (or edge) effects. Finally, the eigenvalues and eigenfunctions of the Hamiltonian are calculated using the Arnoldi method combined with Dirichlet boundary conditions. form:

In this framework, the VBM parameters take the following

$$
\begin{gathered}
|\beta|=\sqrt{\frac{I_{1 / 2}}{I_{3 / 2}}}, \\
|\gamma|=\sqrt{\frac{I_{-1 / 2}}{I_{3 / 2}},}
\end{gathered}
$$

with $I_{j}=\int\left|\phi_{j}^{3 / 2}(\mathbf{r})\right|^{2} d \mathbf{r}$. The VBM parameters being small, the definition given here corresponds to the definition given in Sec. II A. The maximum value of $\theta$ is evaluated when the highest edge of the QD reaches the highest point of the deformed nanohole [see Fig. 1(c)]. We then find $\theta_{\max } \sim 15^{\circ}$.

Figure 9 shows the evolution of $|\beta|$ and $|\gamma|$ as a function of $\theta$ for different in-plane anisotropy $\epsilon \in\{0,0.3,0.5\}$. The height of the QD varies from $h_{\mathrm{QD}}=2$ to $6 \mathrm{~nm}$, while keeping the same radius $R_{\mathrm{QD}}=30 \mathrm{~nm}$. Note that $h_{\mathrm{QD}}$ and $R_{\mathrm{QD}}$ are defined in Fig. 3(a). We observe that $|\gamma|$ is quasi-independent of the global geometry of the dot, but it depends only on the 

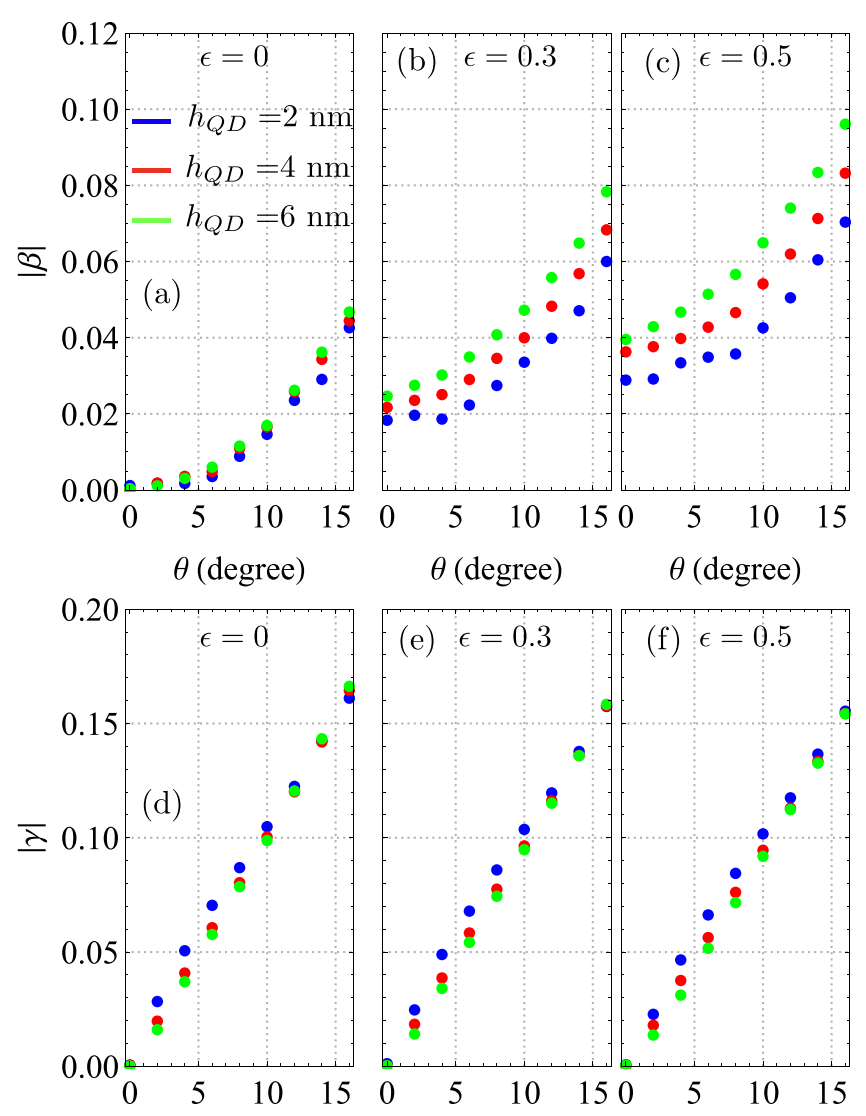

FIG. 9. Quantitative estimations of $|\beta|$ (a)-(c) and $|\gamma|$ (d)-(f) as a function of different QD geometries, with $\epsilon=\{0,0.3,0.5\}$, and $\theta$ varies from 0 to $\theta_{\max }$ (see the text). The radius of the QD is kept constant, $R_{\mathrm{QD}}=30 \mathrm{~nm}$, while the height takes the values 2,4 , and $6 \mathrm{~nm}$.

out-of-plane distortion $\theta$, as demonstrated in Sec. II C. $|\beta|$ depends on the size, but also on the asymmetries of the dot. The trends shown in Fig. 9 are in agreement with the toy model, and they indicate that a modest symmetry breaking of a $C_{2 v}$-potential leads to significant VBM parameter amplitudes enhancing the DE emission, in agreement with experimental results.

\section{APPENDIX C: ASYMPTOTIC BEHAVIOR OF THE CIRCULAR POLARIZATION WITH THE LONGITUDINAL MAGNETIC FIELD}

We provide in this Appendix a detailed explanation of the asymptotic behavior of the circular polarization degree on both sides of the anticrossing, in addition to the fully numerical treatment shown in Fig. 5(c). We focus here on the branches $X_{2}$ and $X_{3}$ for which the anticrossing develops. The diagonalization of the total Hamiltonian gives for the four branches $X_{i}$ the representative states $\left|X_{i}\right\rangle=$ $\sum_{j \in\{+1,-1,+2,-2\}} a_{j}^{i}|j\rangle$, where $a_{j}^{i}$ are continuous functions of the magnetic field. For simplicity, we neglect the role of $\beta$ considering that the $\{|j\rangle\}$ set is the heavy-hole $e-h$ pair basis. As the experimental conditions leads to an equi-repartition of the populations (off-resonant excitation), $\rho_{c}^{X_{i}}$ is equal to $\frac{\left|a_{+1}^{i}\right|^{2}-\left|a_{-1}^{i}\right|^{2}}{\left|a_{+1}^{i}\right|^{2}+\left|a_{-1}^{i}\right|^{2}} \cdot \rho_{c}^{X_{i}}$ becomes analytically tractable (i) when considering $\tilde{\Delta} \sim \Delta_{12}, \tilde{\Delta} \sim-\Delta_{11}$, and $\tilde{\Delta} \ll \Delta_{0}$; and (ii) when regarding its asymptotic dependence with the magnetic field $B_{z}$ far from the anticrossing region, i.e., $B_{z} \ll B_{\mathrm{AC}}$ and $B_{z}>B_{\text {AC. }}$

For the branch $X_{2}$, we find

$$
\begin{gathered}
\rho_{c}^{X_{2}} \sim-\delta_{B} / \Delta_{1} \text { for } B_{z} \ll B_{\mathrm{AC}}, \\
\rho_{c}^{X_{2}} \sim \frac{-2 \delta_{B}\left(2 \Delta_{0}-\delta_{D}\right)}{4 \Delta_{0}^{2}-4 \delta_{D} \Delta_{0}+\delta_{D}^{2}+\delta_{B}^{2}} \text { for } B_{z}>B_{\mathrm{AC}},
\end{gathered}
$$

and finally for the branch $X_{3}$ we find

$$
\begin{gathered}
\rho_{c}^{X_{3}} \sim-\frac{\delta_{B}}{\Delta_{0}-\Delta_{1} / 2} \text { for } B_{z} \ll B_{\mathrm{AC}}, \\
\rho_{c}^{X_{3}} \sim-1+\frac{2 \Delta_{1}^{2}}{\Delta_{1}^{2}+\left(2 \delta_{B}\right)^{2}} \text { for } B_{z}>B_{\mathrm{AC}}
\end{gathered}
$$

with $\delta_{B}=g_{B}^{z} \mu_{B} B_{z}$ and $\delta_{D}=g_{D}^{z} \mu_{B} B_{z}$ being the Zeeman energy of the bright and dark excitons, respectively.

We note that the expressions for $\rho_{c}^{X_{2}}\left(B_{z} \ll B_{\mathrm{AC}}\right)$ and $\rho_{c}^{X_{3}}\left(B_{z} \gg B_{\mathrm{AC}}\right)$ depend only on the characteristics of the BE, i.e., $g_{B}$ and $\Delta_{1}$, confirming that $X_{2}$ in the low magnetic field regime and $X_{3}$ for high magnetic field are dominated by a strong $\mathrm{BE}$ character. Moreover, the coupling $\tilde{\Delta}$ does not appear explicitly in the expressions $\rho_{c}^{X_{2}}\left(B_{z}>B_{\mathrm{AC}}\right)$ and $\rho_{c}^{X_{3}}\left(B_{z} \ll B_{\mathrm{AC}}\right)$ as a consequence of the linearization used for the evaluation of $\left|a_{ \pm 1}^{j}\right|^{2}$.

The model can now be compared to the experimental results shown in Fig. 5(c). First, for the branch $X_{2}$, the sign inversion of $\rho_{c}^{X_{2}}$ for $B>B_{\mathrm{AC}}$ is described by the asymptotic expressions $(\mathrm{C} 1)$ and $(\mathrm{C} 2)$. The denominator in $(\mathrm{C} 2)$ being always positive, $\rho_{c}^{B 2}$ will become negative at some critical field $B$ provided that $g_{D}^{z}>0$, i.e., once $g_{D}^{z} \mu_{B} B \geqslant 2 \Delta_{0}$. This predicts the sign inversion for a magnetic field close to $\sim 2 \Delta_{0} / g_{D}^{z} \mu_{B}=2.7 \mathrm{~T}$, in reasonable agreement with the experimental data.

Secondly, the expression of $\rho_{c}^{X_{3}}$ for the branch $X_{3}$ predicts no singularities through the anticrossing, unlike $X_{2}$.

Lastly, we observe experimentally in Fig. 5(c), in the low magnetic field regime, that the decrease of $\rho_{c}^{X_{2}}$ is slightly larger than that of $\rho_{c}^{X_{3}}$. Our model also accounts for this trend since, in absolute value, the slope of the branch $X_{2}$ is $1 / \Delta_{1}$ according to Eq. (C1), which is larger than the $X_{3}$ slope, $1 /\left(\Delta_{0}-\Delta_{1} / 2\right)$, deduced from Eq. (C3).

This simple model, in combination with values obtained from fits to the Zeeman spectroscopy data, explains well the sign inversion of circular polarization in $X_{2}$ and the lack of sign inversion in $X_{3}$, confirming the presence of the darkbright anticrossing.
[1] T. Heindel, A. Thoma, I. Schwartz, E. R. Schmidgall, L. Gantz, D. Cogan, M. Strauß, P. Schnauber, M. Gschrey, J.-H. Schulze,
A. Strittmatter, S. Rodt, D. Gershoni, and S. Reitzenstein, APL Photon. 2, 121303 (2017). 
[2] I. Schwartz, E. R. Schmidgall, L. Gantz, D. Cogan, E. Bordo, Y. Don, M. Zielinski, and D. Gershoni, Phys. Rev. X 5, 011009 (2015).

[3] B. Siarry, B. Eble, F. Bernardot, P. Grinberg, C. Testelin, M. Chamarro, and A. Lemaître, Phys. Rev. B 92, 155315 (2015).

[4] B. Eble, C. Testelin, P. Desfonds, F. Bernardot, A. Balocchi, T. Amand, A. Miard, A. Lemaître, X. Marie, and M. Chamarro, Phys. Rev. Lett. 102, 146601 (2009).

[5] F. Fras, B. Eble, B. Siarry, F. Bernardot, A. Miard, A. Lemaître, C. Testelin, and M. Chamarro, Phys. Rev. B 86, 161303(R) (2012).

[6] D. Cogan, O. Kenneth, N. H. Lindner, G. Peniakov, C. Hopfmann, D. Dalacu, P. J. Poole, P. Hawrylak, and D. Gershoni, Phys. Rev. X 8, 041050 (2018).

[7] I. Schwartz, D. Cogan, E. R. Schmidgall, Y. Don, L. Gantz, O. Kenneth, N. H. Lindner, and D. Gershoni, Science 354, 434 (2016).

[8] S.-Y. Shiau, B. Eble, V. Voliotis, and M. Combescot, Phys. Rev. B 101, 161405(R) (2020).

[9] S. Lüker, T. Kuhn, and D. E. Reiter, Phys. Rev. B 92, 201305(R) (2015).

[10] M. Holtkemper, G. F. Quinteiro, D. E. Reiter, and T. Kuhn, Phys. Rev. Res. 3, 013024 (2021).

[11] M. Neumann, F. Kappe, T. K. Bracht, M. Cosacchi, T. Seidelmann, V. M. Axt, G. Weihs, and D. E. Reiter, Phys. Rev. B 104, 075428 (2021).

[12] Y. Léger, L. Besombes, L. Maingault, and H. Mariette, Phys. Rev. B 76, 045331 (2007).

[13] Y. H. Huo, V. Křápek, O. G. Schmidt, and A. Rastelli, Phys. Rev. B 95, 165304 (2017).

[14] A. Ulhaq, Q. Duan, E. Zallo, F. Ding, O. G. Schmidt, A. I. Tartakovskii, M. S. Skolnick, and E. A. Chekhovich, Phys. Rev. B 93, 165306 (2016).

[15] T. Smoleński, T. Kazimierczuk, M. Goryca, T. Jakubczyk, L. Kłopotowski, L. Cywiński, P. Wojnar, A. Golnik, and P. Kossacki, Phys. Rev. B 86, 241305(R) (2012).

[16] M. Zieliński, Y. Don, and D. Gershoni, Phys. Rev. B 91, 085403 (2015).

[17] S. Germanis, P. Atkinson, R. Hostein, C. Gourdon, V. Voliotis, A. Lemaître, M. Bernard, F. Margaillan, S. Majrab, and B. Eble, Phys. Rev. B 98, 155303 (2018).

[18] M. Zieliński, Phys. Rev. B 103, 155418 (2021).

[19] S. Germanis, P. Atkinson, R. Hostein, S. Suffit, F. Margaillan, V. Voliotis, and B. Eble, Phys. Rev. B 102, 035406 (2020).

[20] J.-W. Luo, G. Bester, and A. Zunger, Phys. Rev. B 92, 165301 (2015).

[21] Y.-H. Liao, C.-C. Liao, C.-H. Ku, Y.-C. Chang, S.-J. Cheng, M. Jo, T. Kuroda, T. Mano, M. Abbarchi, and K. Sakoda, Phys. Rev. B 86, 115323 (2012).

[22] M. Zocher, C. Heyn, and W. Hansen, J. Appl. Phys. 125, 025306 (2019).

[23] P. Atkinson, E. Zallo, and O. G. Schmidt, J. Appl. Phys. 112, 054303 (2012).

[24] Y. H. Huo, A. Rastelli, and O. G. Schmidt, Appl. Phys. Lett. 102, 152105 (2013).

[25] D. P. Miller, J. G. Harper, and T. R. Perry, J. Electrochem. Soc. 108, 1123 (1961).

[26] D. Fuster, Y. González, and L. González, Nanoscale Res. Lett. 9, 309 (2014).
[27] C. Tonin, R. Hostein, V. Voliotis, R. Grousson, A. Lemaitre, and A. Martinez, Phys. Rev. B 85, 155303 (2012).

[28] M. Świderski and M. Zieliński, Phys. Rev. B 100, 235417 (2019).

[29] T. Belhadj, T. Amand, A. Kunold, C.-M. Simon, T. Kuroda, M. Abbarchi, T. Mano, K. Sakoda, S. Kunz, X. Marie et al., Appl. Phys. Lett. 97, 051111 (2010).

[30] This is particularly true for $\Delta_{11}$.

[31] L. Monniello, C. Tonin, R. Hostein, A. Lemaitre, A. Martinez, V. Voliotis, and R. Grousson, Phys. Rev. Lett. 111, 026403 (2013).

[32] A. Reigue, J. Iles-Smith, F. Lux, L. Monniello, M. Bernard, F. Margaillan, A. Lemaitre, A. Martinez, D. P. S. McCutcheon, J. Mørk, R. Hostein, and V. Voliotis, Phys. Rev. Lett. 118, 233602 (2017).

[33] A. Lafuente-Sampietro, H. Utsumi, M. Sunaga, K. Makita, H. Boukari, S. Kuroda, and L. Besombes, Phys. Rev. B 97, 155301 (2018).

[34] M. Bayer, G. Ortner, O. Stern, A. Kuther, A. A. Gorbunov, A. Forchel, P. Hawrylak, S. Fafard, K. Hinzer, T. L. Reinecke, S. N. Walck, J. P. Reithmaier, F. Klopf, and F. Schäfer, Phys. Rev. B 65, 195315 (2002).

[35] E. A. Chekhovich, A. B. Krysa, M. S. Skolnick, and A. I. Tartakovskii, Phys. Rev. Lett. 106, 027402 (2011).

[36] G. Ragunathan, J. Kobak, G. Gillard, W. Pacuski, K. Sobczak, J. Borysiuk, M. S. Skolnick, and E. A. Chekhovich, Phys. Rev. Lett. 122, 096801 (2019).

[37] Actually, the weight of the bright component in the (AS) DE state is proportional to $\left(\Delta_{11}+\Delta_{12}\right) /\left(\Delta_{0}-\Delta_{1} / 2\right)$, which is very weak as $\Delta_{11} \sim-\Delta_{12}$.

[38] G. Sallen, A. Tribu, T. Aichele, R. André, L. Besombes, C. Bougerol, S. Tatarenko, K. Kheng, and J. P. Poizat, Phys. Rev. B 80, 085310 (2009).

[39] L. Monniello, A. Reigue, R. Hostein, A. Lemaitre, A. Martinez, R. Grousson, and V. Voliotis, Phys. Rev. B 90, 041303(R) (2014).

[40] A. Reigue, R. Hostein, and V. Voliotis, Semicond. Sci. Technol. 34, 113001 (2019).

[41] H. Kamada and T. Kutsuwa, Phys. Rev. B 78, 155324 (2008).

[42] M. Anderson, T. Müller, J. Skiba-Szymanska, A. B. Krysa, J. Huwer, R. M. Stevenson, J. Heffernan, D. A. Ritchie, and A. J. Shields, Appl. Phys. Lett. 118, 014003 (2021).

[43] G. Cassabois and R. Ferreira, C. R. Phys. 9, 830 (2008).

[44] K. Peng, S. Wu, J. Tang, F. Song, C. Qian, S. Sun, S. Xiao, M. Wang, H. Ali, D. A. Williams, and X. Xu, Phys. Rev. Appl. 8, 064018 (2017).

[45] M. Zieliński, Phys. Rev. B 102, 245423 (2020).

[46] S. Marcet, K. Ohtani, and H. Ohno, Appl. Phys. Lett. 96, 101117 (2010).

[47] A. Boyer de la Giroday, A. J. Bennett, M. A. Pooley, R. M. Stevenson, N. Sköld, R. B. Patel, I. Farrer, D. A. Ritchie, and A. J. Shields, Phys. Rev. B 82, 241301(R) (2010).

[48] L. Zhai, M. C. Löbl, G. N. Nguyen, J. Ritzmann, A. Javadi, C. Spinnler, A. D. Wieck, A. Ludwig, and R. J. Warburton, Nat. Commun. 11, 4745 (2020).

[49] G. K. Svendsen, J. Skaar, H. Weman, and M.-A. Dupertuis, Phys. Rev. B 92, 205303 (2015). 